\title{
Ultrafast imaging of plasmons in a transmission electron microscope
}

Tom T. A. Lummen, Gabriele Berruto, Andrea Toma, Raymond J. Lamb, Damien McGrouther, et al.

Tom T. A. Lummen, Gabriele Berruto, Andrea Toma, Raymond J. Lamb, Damien McGrouther, Fabrizio Carbone, "Ultrafast imaging of plasmons in a transmission electron microscope," Proc. SPIE 9746, Ultrafast Phenomena and Nanophotonics XX, 974610 (14 March 2016); doi: 10.1117/12.2212404 


\title{
Ultrafast imaging of plasmons in a transmission electron microscope
}

\author{
Tom. T.A. Lummen*a, Gabriele Berruto ${ }^{\mathrm{a}}$, Andrea Toma ${ }^{\mathrm{b}}$, Raymond J. Lamb ${ }^{\mathrm{c}}$, \\ Damien McGrouther ${ }^{c}$, Fabrizio Carbone ${ }^{\mathrm{a}}$ \\ ${ }^{a}$ Laboratory for Ultrafast Microscopy and Electron Scattering (LUMES), ICMP, \\ École Polytechnique Fédérale de Lausanne, CH-1015 Lausanne, Switzerland; \\ ${ }^{\mathrm{b}}$ Italian Institute of Technology (ITT), I-16163 Genova, Italy; \\ ${ }^{\mathrm{c}} \mathrm{SUPA}$, School of Physics and Astronomy, University of Glasgow, \\ Glasgow G12 8QQ, United Kingdom
}

\begin{abstract}
Miniaturized plasmonic and photonic integrated circuits are generally considered as the core of future generations of optoelectronic devices, due to their potential to bridge the size-compatibility gap between photonics and electronics. However, as the nanoscale is approached in increasingly small plasmonic and photonic systems, experimentally observing their behavior involves ever more stringent requirements in terms of both temporal and spatial resolution. This talk focuses on the use of time-resolved Photon-Induced Near-Field Electron Microscopy (PINEM) to study the excitation, propagation, (self-)interference and dynamics of surface plasmon polaritons (SPPs) in various plasmonic nanostructures with both nanometer and ultrafast resolution in a transmission electron microscope. Using this field-ofview technique, we directly show how photo-excited plasmonic interference patterns are controlled through the combination of excitation polarization and nanostructure geometry. Moreover, we capture the propagation of the photoinduced self-interfering plasmonic wave, clearly demonstrating the effects of axial confinement in nanostructured plasmonic thin film stacks.
\end{abstract}

Keywords: ultrafast electron microscopy, PINEM, surface plasmon polaritons, plasmonic interference

\section{INTRODUCTION}

As the nanoscale is approached in increasingly small plasmonic and photonic circuits, important functionalities such as light guiding, bending, splitting and filtering, or processes such as higher harmonic and/or frequency comb generation face ever more stringent requirements in terms of materials, design and, most of all, scientific understanding ${ }^{1-3}$. The latter in particular is of primary importance, as the behavior of light confined to length scales smaller than its wavelength defines a scientific territory that has started to be explored only in recent years. Most promising in this area are so-called Surface Plasmon Polaritons (SPPs) ${ }^{4,5}$, which can be described as propagating electromagnetic fields that are confined to a metallic surface or interface on a sub-wavelength scale ${ }^{1}$. Due to their nature as coupled photon-plasmon quasiparticles, SPPs inherently link photonics and electronics, and as such they are generally envisioned as the information carriers of future integrated circuitry. At present, however, there are still many unanswered questions concerning sub-wavelength optical systems, such that there is a particular need to clarify the behavior of SPPs in simple systems, especially in the experimental domain.

\subsection{Probing evanescent optical fields}

Due to its evanescent, surface-confined nature, the plasmonic near-field of SPPs is only experimentally accessible through localized probes, with conventional imaging methods based on Scanning Near-field Optical Microscopy $(\mathrm{SNOM})^{6,7}$ and other local physical probe methodologies. Unfortunately, this class of tip-based, scanning probe techniques inherently suffer from fundamental limitations in terms of field of view (necessary point-by-point probing), acquisition time (time-intensive scanning and integration), spatial resolution (probe-dimension limited) and time resolution (typically, only static fields can be probed). Moreover, as the physical probe needs to be in the direct proximity of the near-field under study, accessing the evanescent near-field confined to a buried interface is not possible.

*tom.lummen@epfl.ch; phone +41 21693 5336; fax 41216935875

Ultrafast Phenomena and Nanophotonics XX, edited by Markus Betz, Abdulhakem Y. Elezzabi, Proc. of SPIE Vol. 9746, 974610 · ( ) 2016 SPIE · CCC code: 0277-786X/16/\$18

doi: $10.1117 / 12.2212404$

Proc. of SPIE Vol. 9746 974610-1 


\subsection{An electron microscopy approach}

Here, we adopt an entirely different experimental approach, based on Ultrafast Transmission Electron Microscopy (UTEM) methodology, which allows for the study evanescent optical fields in nanostructures by directly imaging their spatial extent, propagation and temporal evolution with nanometer $(\mathrm{nm})$ and ultrafast (few-hundred-femtosecond (fs)) resolution. In particular, we employ Photon-Induced Near-Field Electron Microscopy (PINEM) ${ }^{8,9}$, in which a fs light pulse induces an evanescent field on a nanostructure, which then exchanges photon-energy quanta with an overlapping pulse of imaging electrons. By analyzing the resulting electron distribution using an advanced electron energy analyzer, one can extract the spectral signatures of the energy exchange as a function of relative delay between the two pulses (Fig 1a). Generally, the durations of the optical pump and electron probe pulses (few-hundred-fs) are much longer than the typical lifetime of the photo-induced near-field (on the order of tens of fs), such that the temporal signature essentially corresponds to a convolution of the pump and probe pulses.

The electron distribution at any delay within this temporal overlap reveals the presence of a near-symmetric distribution of sidebands, which develop at the expense of spectral density in the zero-loss peak (ZLP) (See Fig 1b). These equidistant energy gain and loss peaks correspond to different orders of photon-assisted net energy-quantum exchanges, resulting from the complex evolution undergone by the electrons during their interaction with the plasmonic near-field. ${ }^{10}$ Importantly, the degree of energy exchange is proportional to the strength of the plasmonic near-field, as specifically, its component along the electron propagation direction.

This means that by energy-filtering the transmitted electron beam after it has interacted with the sample, leaving only the 'inelastic electrons' to form a so-called PINEM image, one can directly visualize the electromagnetic near-field strength on the nanostructure, as is sketched for a silver nanowire in Figure 1c. This method has recently been used to image the evanescent fields in a multitude of nanostructures, including carbon nanotubes ${ }^{8}$, metallic nanoparticles ${ }^{11,12}$ and nanoantennas ${ }^{8,13,14}$, and even in biological systems such as stained bacterial cells and protein vesicles ${ }^{15}$.

Since the PINEM methodology inherently adopts a transmission geometry, we employ it here to directly film the dynamical evolution of a photo-induced self-interfering plasmonic wave propagating at a buried interface. Thus, we extract the propagation speed of the corresponding photo-excited SPP waves. Furthermore, we show that the spatial distribution of the observed photo-induced plasmonic interference can be controlled through the combination of excitation polarization and nanostructure design.
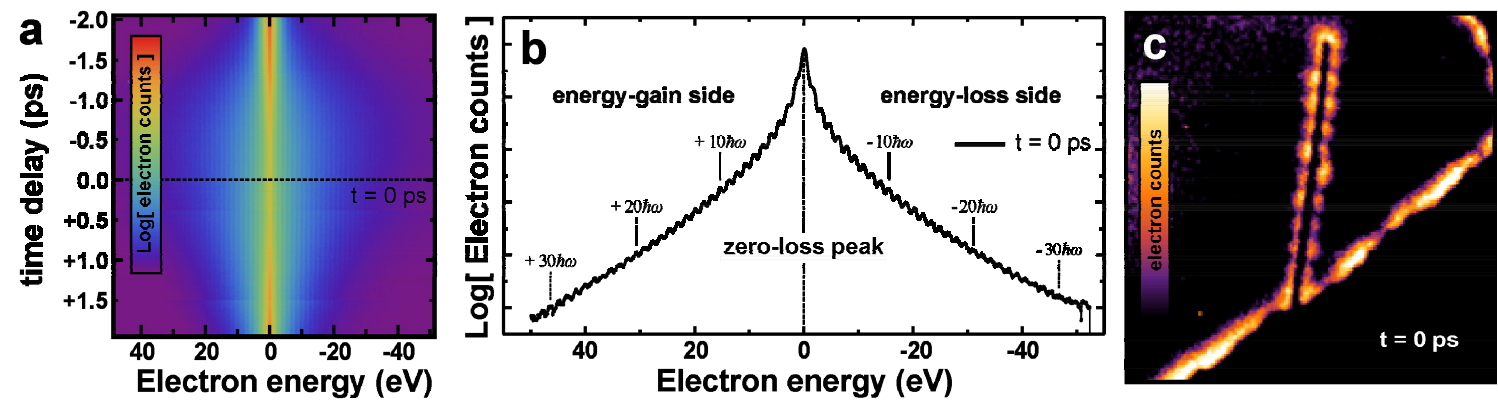

Figure 1. a. PINEM time delay scan of electron energy spectra, showing the inelastic interactions between photo-electrons in the probe and the plasmonic near-field photo-induced on a silver nanowire around zero delay $(t=0 \mathrm{ps})$. $b$. Electron energy spectrum at $\mathrm{t}=0 \mathrm{ps}$, showing the net exchange of over 30 photon quanta $\hbar \omega$. c. Energy-filtered PINEM image of a single silver nanowire suspended off a standard TEM grid at $t=0 \mathrm{ps}$. This image is formed by selecting out only the electrons on the energy gain side of the zero-loss peak, and spatially resolving their distribution.

\section{EXPERIMENTAL}

\subsection{Materials}

A thin silver film $(\approx 30 \mathrm{~nm})$ was sputtered onto a custom-made $\mathrm{Si}_{3} \mathrm{~N}_{4}$ substrate using a sputter coater, and nanoscale cavities (NCs) were written into the Ag layer using a raster-scanned focused-ion beam (FIB). The sample (see Figure 2) was kept in low oxygen conditions and measured in the UTEM at EPFL (at a temperature of $295 \mathrm{~K}$ and a pressure of $\approx$ $10^{5} \mathrm{~Pa}$ ) within a week of patterning. For the propagation experiment, nominal NC widths were $250 \mathrm{~nm}$ (calibrated widths $\approx 274 \mathrm{~nm}$ ), with nominal NC lengths incrementing from 300 to $5000 \mathrm{~nm}$ in $100 \mathrm{~nm}$ steps (from left to right, NCs in field 
of view in Figure 4a, calibrated lengths $( \pm 0.3 \%)$ : 698, 809, 919, 982, 1113, 1175, and $1273 \mathrm{~nm})$. PINEM image calibration was done using the calibrated $\mathrm{NC}$ lengths in the field of view as independent references, with the final $\mathrm{nm}$ per-pixel parameter taken as the mean of the calibration factors independently determined for the different NCs. The resulting uncertainty in the image scale is the principal contribution to the error bars on the extracted SPP group velocity $\left(\mathrm{v}_{\mathrm{g}}\right)$.

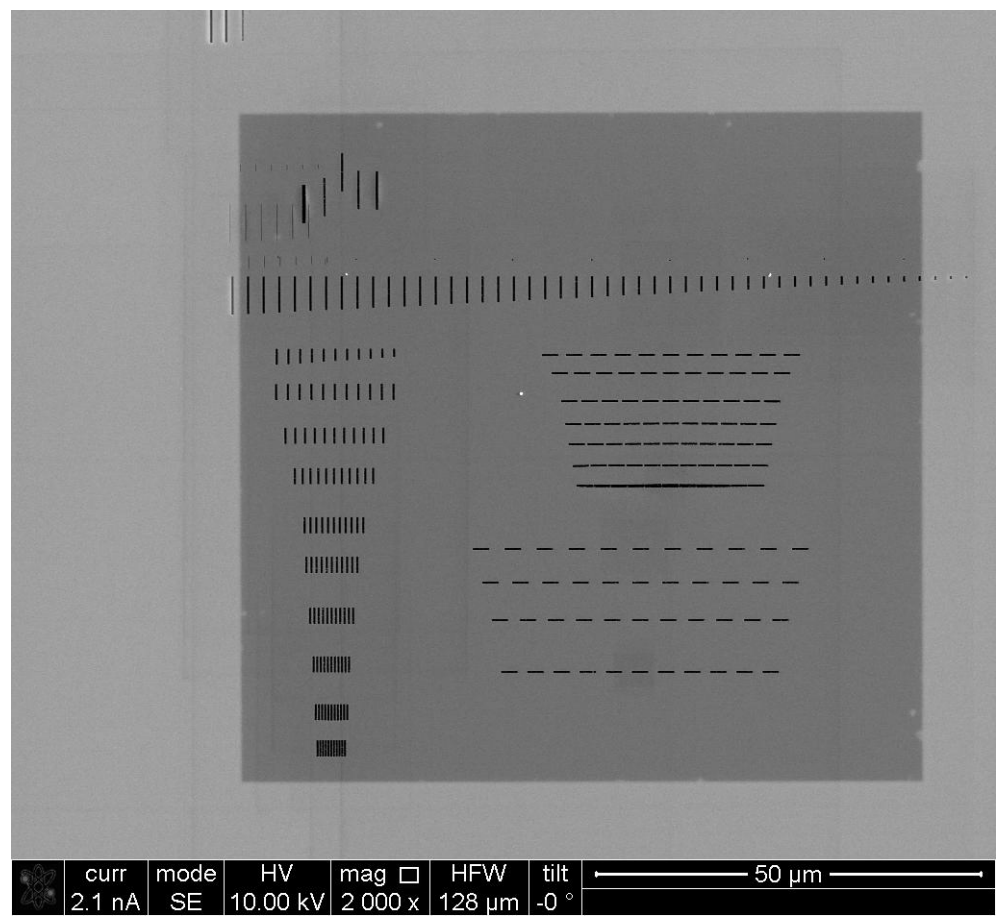

Figure 2. Scanning Electron Microscope image of the nano-patterned silver film on a custom-made $\mathrm{Si}_{3} \mathrm{~N}_{4}$ substrate.

\subsection{Apparatus}

A $300 \mathrm{kHz}$ train of linearly polarized light pulses (pulse duration 80 fs (full-width-at-half-maximum (FWHM) from autocorrelation), $\lambda_{0}=786 \mathrm{~nm}$ ) was split to yield two beams, one of which was tripled in frequency using standard nonlinear conversion techniques, in order to generate a few-nJ UV pulse train $(\lambda=262 \mathrm{~nm})$. This UV pulse train was then delivered to a custom truncated-cone LaB6 photo-cathode (15 $\mu \mathrm{m}$ diameter truncation plane, AP-Tech) in a custommodified ultrafast transmission electron microscope (UTEM) ${ }^{13}$. The second optical beam was routed through a variable optical delay line and weakly focused on the sample in the UTEM at near-normal incidence. The beam focus was approximately $100 \mu \mathrm{m}$ in diameter at the sample plane, such that the electric field of the pump beam was uniform across the field of view of the electron beam. Optical pump fluences employed were of the order of 1 to $3 \mathrm{~mJ} \mathrm{~cm}^{-2}$, corresponding to an estimated peak excitation energy density of about $30 \mathrm{GW} \mathrm{cm}^{-2}$. Under these experimental conditions electron-probe excitation of SPPs is much weaker than photo-excitation of SPPs, and the former is thus considered entirely negligible ${ }^{16}$.

The custom-modified JEOL JEM 2100 microscope is schematically depicted in Figure 3. A more detailed description and characterization of the system can be found in Ref. 13. For the PINEM experiments described in this work, the UTEM was operated at $200 \mathrm{keV}$ in photoelectron mode. The GIF imaging camera was operated with a 0.05 eV-per-channel dispersion setting, with typical exposure times of $60 \mathrm{~s}$ for energy-filtered images and $10 \mathrm{~s}$ for energy spectra. Electron energy loss spectra were aligned based on their zero loss peak (ZLP) positions using a differentialbased maximum intensity alignment algorithm. 


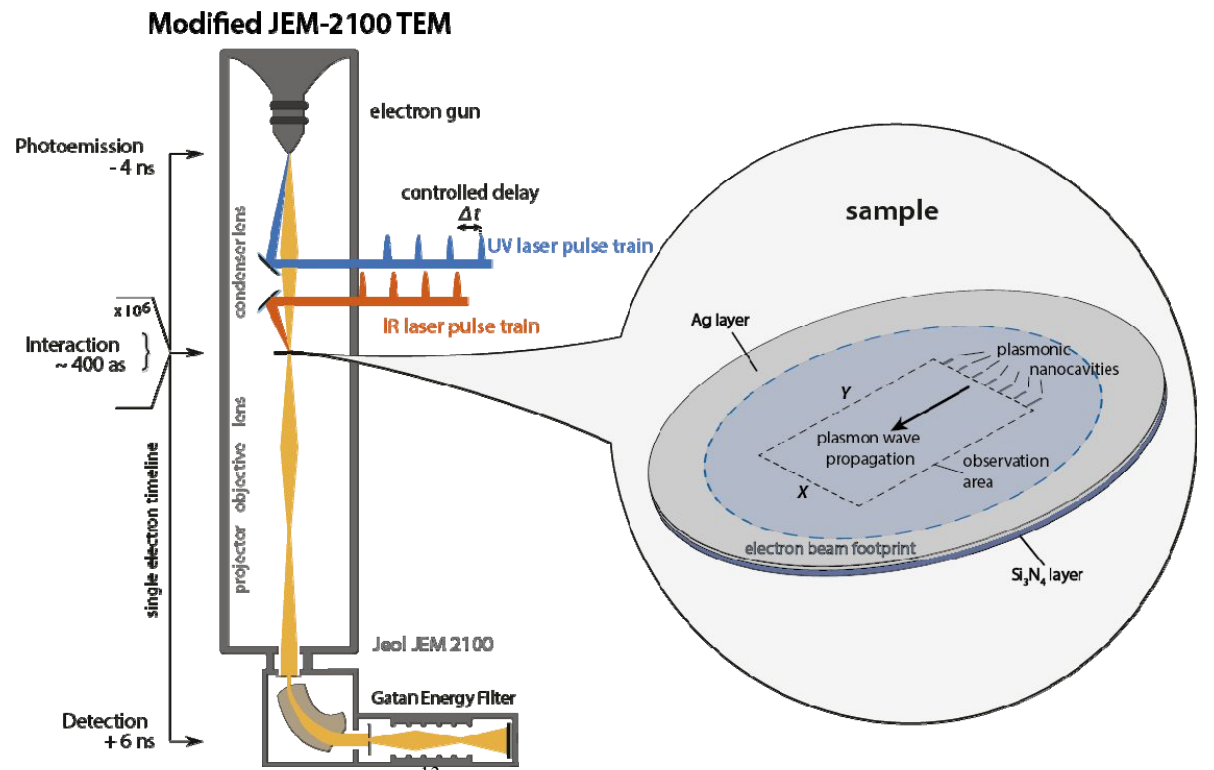

Figure 3. Schematic of the custom-modified UTEM ${ }^{13}$. Variably delayed light (pump) and photo-electron (probe) pulses are spatially overlapped on the nano-patterned $\mathrm{Ag}$-on- $\mathrm{Si}_{3} \mathrm{~N}_{4}$ sample (see zoom-in), after which the resulting electron distribution is analyzed using an advanced energy-filtering detection system.

\section{RESULTS}

\subsection{Filming plasmon propagation}

As sketched in figure 3, both the optical pump and electron probe pulses are incident on the Ag side of the sample. It is well-established that such an incident optical plane wave couples to the edges of metallic nano-cavities (NCs), giving rise to SPPs that are first transmitted through the apertures to the other side of the metallic layer, where they are subsequently launched across the opposing metallic interface. ${ }^{17,18}$ In first approximation, photo-induced SPPs propagate outwards from such a NC source in a pattern akin to that of a point-dipole source. As a result, the SPPs launched from different NCs in a linear array coherently add up to form a self-interfering plasmonic wave propagating away from the array (along the direction indicated in Figure 3). Using PINEM imaging, we follow the formation and propagation of this plasmonic wave in real time, taking snapshots every 50 fs (see Figure 4a). For each time delay, we then divide up the image in a series of horizontal slices at different distances $Y$ from the $\mathrm{NC}$ array, take the spatial Fourier transform of each slice and integrate its singular peak corresponding to the characteristic SPP interference period. By subsequently combining all the data, we obtain a complete time-space map of the dynamic evolution of the plasmonic wave (Figure $4 \mathrm{~b}$ ), which illustrates the SPP propagation. We then fit the thus-constructed time-traces for each position $Y$ with a Gaussian line-shape, and subsequently plot the corresponding temporal center positions in Figure 4c. A linear fit to this dataset directly gives the group velocity of the composite SPP wave, at a value of $(1.01 \pm 0.12) \times 10^{8} \mathrm{~m} \mathrm{~s}^{-1}$.
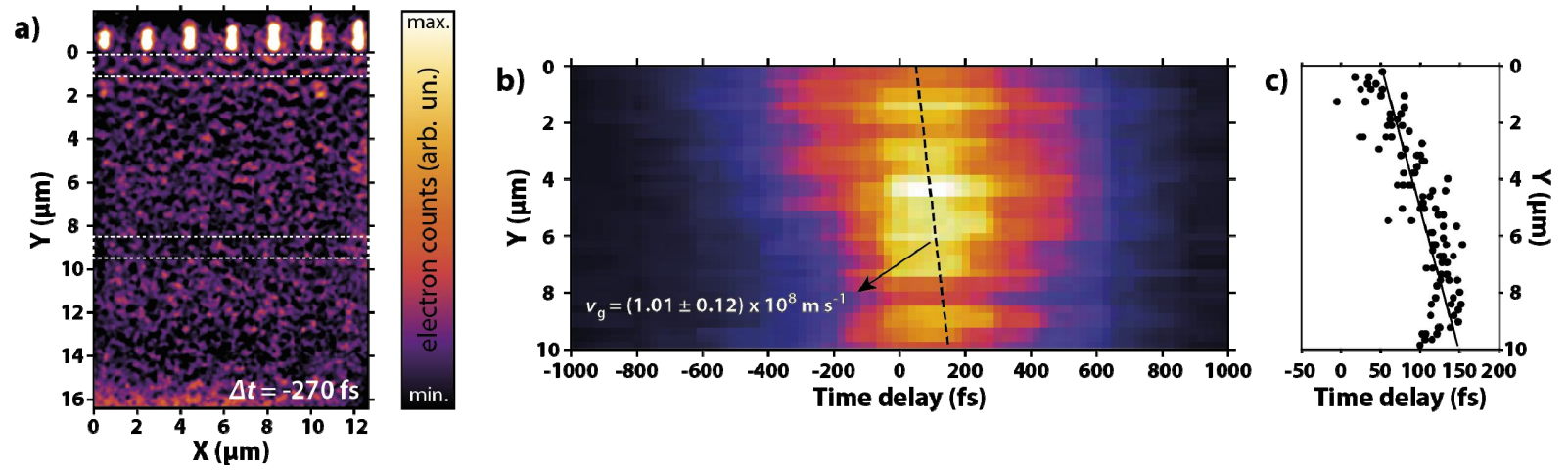

Figure 4. a. Experimental PINEM image of the photo-excited SPP wave propagating at the buried metallic interface $(\Delta t=0)$. White dashed rectangles indicate example horizontal slicing of the image for Fourier analysis. b. Time-space map of SPP 
propagation composed through Fourier analysis (see text). The dashed line depicts the extracted group velocity. c. Center position of the Gaussian-fitted time-traces versus distance traveled by the plasmonic wave. The slope of a linear fit (solid line) corresponds to the plasmonic group velocity.

\subsection{Control of plasmonic interference}

Aside from allowing access to dynamics, the visualization capability of PINEM also holds promise for the design of plasmonic interference patterns. By combining specific nano-pattern geometries with appropriate light polarization for photo-excitation, one can tailor the interferometric structure that is obtained. ${ }^{18,19}$ Furthermore, since the radiation pattern of a single SPP source can be accurately modeled by that of a single dipole emitter (whose form is analytically known), it becomes possible to predict photo-induced plasmonic interference patterns beforehand.

In a demonstration of this approach, we both calculated and measured the plasmonic interference pattern generated by two linear NC arrays placed in a specific relative geometry, under a given linearly polarized photoexcitation (Figure 5). The calculated and experimental interference patterns are in excellent qualitative agreement, which underlines the potential of the concept. In this specific geometry, the standing wave pattern that is set up by counterpropagating SPPs launched from the different NC arrays features several regular arrays of nanoscale 'islands' of nearfield intensity. Because of the coupled plasmon-polariton nature of SPPs, such structures thus also correspond to photoinduced regular arrays of charge density in the metal film. These results shows that is indeed possible to control the spatial distribution of plasmonic interference patterns, and thus charge density, in nanostructure thin metal films.
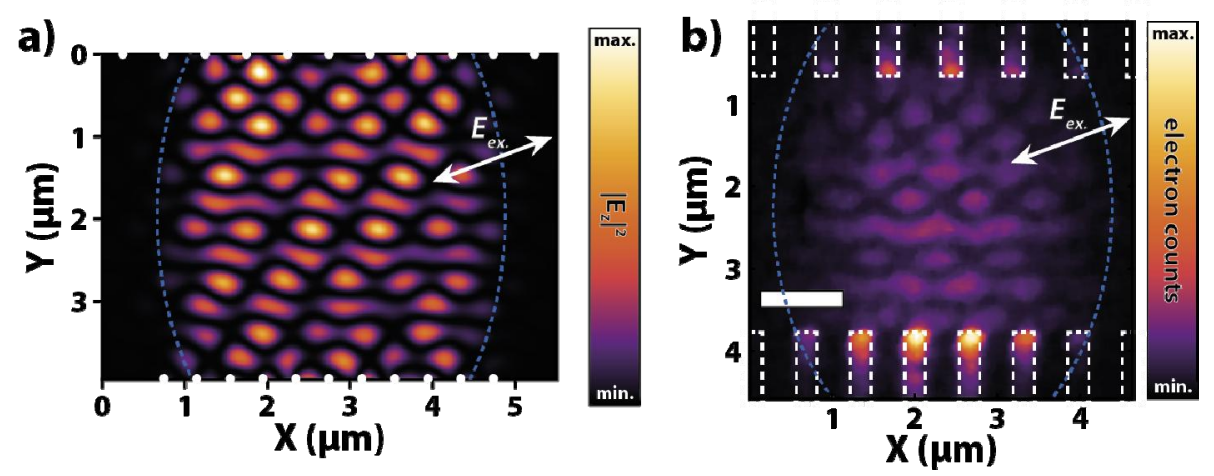

Figure 5. a. Analytically model of the coherent superposition of the SPPs launched from two opposing linear arrays of pointdipole sources, yielding a complex plasmonic interference pattern featuring several regular arrays of near-field intensity 'islands'. b. Corresponding experimental PINEM image of the same structure, showing a plasmonic interference pattern with very similar features. White dashed rectangles indicate NCs. In both panels, the dashed blue line outlines the electron beam footprint, while the double-headed white arrow indicates the polarization of the excitation light.

\section{SUMMARY}

To summarize, in this paper we utilize ultrafast electron microscopy, PINEM in particular, to directly visualize photoexcited plasmonic interference patterns in a nanostructured Ag thin film. We show that using the PINEM technique, it is possible to directly measure the speed of interfering SPPs that propagate at a buried metallic interface. Furthermore, we demonstrate that it is possible to control the spatial distribution of photo-induced plasmonic interference patterns through the combination of tailored nano-structuring and appropriate excitation polarization, and that such patterns can be qualitatively modeled using simple point-dipole approximations for the SPP sources.

\section{REFERENCES}

[1] Barnes, W. L., Dereux, A., and Ebbesen, T.W., "Surface plasmon subwavelength optics," Nature 424, 824-830 (2003).

[2] Ozbay, E. "Plasmonics: Merging Photonics and Electronics at Nanoscale Dimensions," Science 311, 189-193 (2006). 
[3] Prodan, E., Radloff, C., Halas, N.J., and Nordlander, P., "A Hybridization Model for the Plasmon Response of Complex Nanostructures," Science 302, 419-422 (2003).

[4] Maier, S.A., and Atwater, H.A., "Plasmonics: Localization and guiding of electromagnetic energy in metal/dielectric structures," J. Appl. Phys. 98, 011101 (2005).

[5] De Leon, N.P., Shields, B.J., Yu, C.L., et al., "Tailoring Light-Matter Interaction with a Nanoscale Plasmon Resonator," Phys. Rev. Lett. 108, 226803 (2012).

[6] Heinzelmann, H., and Pohl, D.W., "Scanning near-field optical microscopy," Appl. Phys. A 59, 89-101 (1994).

[7] Wüest, R., Buchler, B.C., Harbers, R., et al., "Near-field optical microscopy of light propagation through photonic crystal waveguide tapers," Proc. of SPIE 5840, 110-117 (2005).

[8] Barwick, B., Flannigan, D.J., and Zewail, A.H., "Photon-induced near-field electron microscopy," Nature 462, 902-906 (2009).

[9] Barwick, B., and Zewail, A.H., "Photonics and Plasmonics in 4D Ultrafast Electron Microscopy," ACS Photonics 2, 1391-1402 (2015).

[10] García de Abajo, F.J., Asenjo-Garcia, A., and Kociak, M., "Multiphoton Absorption and Emission by Interaction of Swift Electrons with Evanescent Light Fields," Nano Lett. 10, 1859-1863 (2010).

[11] Yurtsever, A., and Zewail, A., "Direct Visualization of Near-Fields in Nanoplasmonics and Nanophotonics," Nano Lett. 12, 3334-3338 (2012).

[12] Yurtsever, A., Baskin, S.J., and Zewail, A., "Entangled Nanoparticles: Discovery by Visualization in 4D Electron Microscopy," Nano Lett. 12, 5027-5032 (2012).

[13] Piazza, L, Masiel, D.J., LaGrange, T., Reed, B.W., Barwick, B., and Carbone, F., "Design and implementation of a fs-resolved transmission electron microscope based on thermionic gun technology," Chem. Phys. 423, 7984 (2013).

[14] Piazza, L., Lummen, T.T.A., Quiñonez, E., Murooka, Y., Reed, B.W., Barwick, B., and Carbone, F., "Simultaneous observation of the quantization and the interference pattern of a plasmonic near-field," Nat. Commun. 6, 6407 (2015).

[15]Flannigan, D.J., Barwick, B., and Zewail, A.H., "Biological imaging with 4D ultrafast electron microscopy," Proc. Natl. Acad. Sci. 107, 9933-9937 (2010).

[16] Park, S. T., Lin, M., and Zewail, A. H., "Photon-induced near-field electron microscopy (PINEM): theoretical and experimental," New J. Phys. 12, 123028 (2010).

[17] Lalanne, P., Hugonin, J.P., and Rodier, J.C., "Approximate model for surface-plasmon generation at slit apertures," J. Opt. Soc. Am. A 23, 1608-1615 (2006).

[18]Lin, J., Mueller, J.P.B., Wang, Q., Yuan, G., Antoniou, N., Yuan, X.-C., and Capasso, F., "PolarizationControlled Tunable Directional Coupling of Surface Plasmon Polaritons," Science 340, 331-334 (2013).

[19] Spektor, G., David, A., Gjonaj, B., Bartal, G., and Orenstein, M., "Metafocusing by a Metaspiral Plasmonic Lens," Nano Lett. 15, 5739-5743 (2015). 